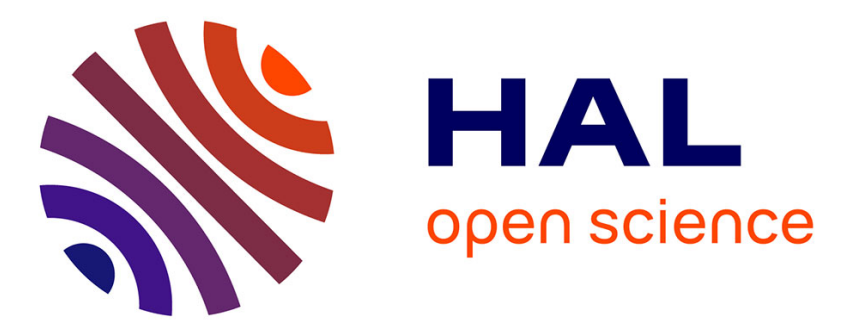

\title{
Stochastic approximation of quasi-stationary distributions for diffusion processes in a bounded domain
}

\author{
Michel Benaïm, Nicolas Champagnat, Denis Villemonais
}

\section{To cite this version:}

Michel Benaïm, Nicolas Champagnat, Denis Villemonais. Stochastic approximation of quasi-stationary distributions for diffusion processes in a bounded domain. Annales de l'Institut Henri Poincaré (B) Probabilités et Statistiques, 2021, 57 (2), pp.726-739. 10.1214/20-AIHP1093 . hal-02101739v3

\section{HAL Id: hal-02101739 \\ https://hal.science/hal-02101739v3}

Submitted on 15 Feb 2021

HAL is a multi-disciplinary open access archive for the deposit and dissemination of scientific research documents, whether they are published or not. The documents may come from teaching and research institutions in France or abroad, or from public or private research centers.
L'archive ouverte pluridisciplinaire HAL, est destinée au dépôt et à la diffusion de documents scientifiques de niveau recherche, publiés ou non, émanant des établissements d'enseignement et de recherche français ou étrangers, des laboratoires publics ou privés. 


\title{
Stochastic approximation of quasi-stationary distributions for diffusion processes in a bounded domain
}

\author{
Michel Benaïm ${ }^{1}$, Nicolas Champagnat ${ }^{2}$, Denis Villemonais ${ }^{2}$
}

February 15, 2021

\begin{abstract}
We study a random process with reinforcement, which evolves following the dynamics of a given diffusion process in a bounded domain and is resampled according to its occupation measure when it reaches the boundary. We show that its occupation measure converges to the unique quasi-stationary distribution of the diffusion process absorbed at the boundary of the domain. Our proofs use recent results in the theory of quasi-stationary distributions and stochastic approximation techniques.
\end{abstract}

\section{Résumé}

Nous étudions un processus stochastique avec renforcement, qui évolue suivant une diffusion dans un domaine borné, avec ré-échantillonnage suivant sa mesure d'occupation lorsqu'il atteint la frontière. Nous montrons que sa mesure d'occupation converge vers l'unique distribution quasistationnaire de la diffusion absorbée au bord du domaine. Nos preuves s'appuient sur des résultats récents en théorie des distributions quasistationnaires et sur des techniques d'approximation stochastique.

Keywords: random processes with reinforcement, stochastic approximation, pseudo-asymptotic trajectories, quasi-stationary distributions.

2010 Mathematics Subject Classification. Primary: 60B12, 60J60, 60B10, 60F99; Secondary: 60J70.

\section{Introduction}

Let $\left(\Omega,\left(\mathcal{F}_{t}\right)_{t \in[0,+\infty)},\left(X_{t}\right)_{t \in[0,+\infty)},\left(\mathbb{P}_{x}\right)_{x \in E \cup\{\partial\}}\right)$ be a time homogeneous Markov process with state space $E \cup\{\partial\}$, where $E$ is a measurable space and $\partial \notin E$ is

\footnotetext{
${ }^{1}$ Université de Neuchâtel, Switzerland

${ }^{2}$ Université de Lorraine, CNRS, Inria, IECL, F-54000 Nancy, France

E-mail: Nicolas.Champagnat@inria.fr, Denis.Villemonais@univ-lorraine.fr
} 
an absorbing state for the process. This means that $X_{s}=\partial$ implies $X_{t}=\partial$ for all $t \geq s, \mathbb{P}_{x}$-almost surely for all $x \in E$ and, in particular,

$$
\tau_{\partial}:=\inf \left\{t \geq 0, X_{t}=\partial\right\}
$$

is a stopping time. We also assume that $\mathbb{P}_{x}\left(\tau_{\partial}<\infty\right)=1$ and $\mathbb{P}_{x}\left(t<\tau_{\partial}\right)>0$ for all $t \geq 0$ and $\forall x \in E$.

We consider a random process $\left(Y_{t}\right)_{t>0}$ with reinforcement, which evolves following the dynamic of $X$ when it lies in $E$ and which is resampled according to its occupation measure when it reaches $\partial$. More precisely, given a probability measure $\mu$ on $E$, we set

$$
Y_{t}=\sum_{k=1}^{\infty} 1_{t \in\left[\theta_{k-1}, \theta_{k}\right)} X_{t-\theta_{k-1}}^{(k)}, \quad \forall t \geq 0,
$$

where $\theta_{0}=0$,

- $\left(X_{t}^{(1)}, t \geq 0\right)$ is a realization of the process $\left(X_{t}, t \geq 0\right)$ with $X_{0}^{(1)} \sim \mu$ (i.e. under $\mathbb{P}_{\mu}$ ) and the stopping time $\theta_{1}$ is defined as $\theta_{1}=\tau_{\partial}^{(1)}$ the first hitting time of $\partial$ by $X^{(1)}$,

- given $X^{(1)},\left(X_{t}^{(2)}, t \geq 0\right)$ is a realization of the process $\left(X_{t}, t \geq 0\right)$ with $X_{0}^{(2)} \sim \mu_{\theta_{1}}$, where

$$
\mu_{\theta_{1}}=\frac{1}{\theta_{1}} \int_{0}^{\theta_{1}} \delta_{Y_{s}} d s
$$

and $\theta_{2}-\theta_{1}=\tau_{\partial}^{(2)}$ the first hitting time of $\partial$ by $X^{(2)}$,

- for all $k \geq 1$, given $X^{(1)}, X^{(2)}, \ldots, X^{(k)},\left(X_{t}^{(k+1)}, t \geq 0\right)$ is a realization of the process $\left(X_{t}, t \geq 0\right)$ with $X_{0}^{(k+1)} \sim \mu_{\theta_{k}}$, where

$$
\mu_{\theta_{k}}=\frac{1}{\theta_{k}} \int_{0}^{\theta_{k}} \delta_{Y_{s}} d s
$$

and $\theta_{k+1}-\theta_{k}=\tau_{\partial}^{(k+1)}$ the first hitting time of $\partial$ by $X^{(k+1)}$.

We also define for all $t \geq 0$

$$
\mu_{t}=\frac{1}{t} \int_{0}^{t} \delta_{Y_{s}} d s, \quad \text { i.e. } \quad \mu_{t}(f)=\frac{1}{t} \int_{0}^{t} f\left(Y_{s}\right) d s, \quad \forall f \in \mathcal{B}_{b}(E) .
$$

This process has been studied in several situations, with the main goal of proving an almost sure convergence result for the occupation measure $\mu_{t}$ when $t \rightarrow+\infty$. In the finite state space case and in a discrete time setting, Aldous, Flannery and Palacios [1] solved this problem by showing that the proportion of colours in a Pólya urn type process converges almost surely to the left eigenfunction of the replacement matrix, which was also identified as the quasi-stationary 
distribution of a corresponding Markov chain (we refer the reader to the surveys $[15,19]$ and to the book [11] for general references on quasi-stationary distributions; basic facts and useful results on quasi-stationary distributions are also reminded in Section 3). Under a similar setting but using stochastic approximation techniques, Benaïm and Cloez [3] and Blanchet, Glynn and Zheng[6] independently proved the almost sure convergence of the occupation measure $\mu_{t}$ toward the quasi-stationary distribution of $X$. These works have since been generalized to the compact state space case by Benaïm, Cloez and Panloup [4] under general criteria for the existence of a quasi-stationary distribution for $X$. Continuous time diffusion processes with smooth bounded killing rate on compact Riemanian manifolds have been recently considered by Wang, Roberts and Steinsaltz [21], who show that a similar algorithm with weights also converges toward the quasi-stationary distribution of the underlying diffusion process. Recently, Mailler and Villemonais [14] have proved such a convergence result for processes with smooth and bounded killing rate evolving in non-compact (more precisely unbounded) spaces using a measure-valued Pólya process representation of this reinforced algorithm.

The aim of the present paper is to solve the question of convergence of the occupation measure toward the quasi-stationary distribution of $X$ when this process is a uniformly elliptic diffusion evolving in an open bounded connected open set $D$ with $C^{2}$ boundary $\partial D$, with hard killing when the process hits the boundary. This answers positively the open problem stated in Section 8 of [4]. Note that the difficulty is twofold: firstly, the state space $E=D$ is an open domain in $\mathbb{R}^{d}$ and is thus non-compact; secondly, the absorption occurs through killing at the boundary, which corresponds to an infinite killing rate.

Our main assumptions concern the $C^{2}$ regularity of the domain and of the parameters of the diffusion $X$. They are satisfied in particular if the coefficients of the stochastic differential equation satisfied by $X$ are Hölder continuous. Our assumptions ensure the existence of a unique quasi-stationary distribution $\alpha$ for $X$ and allows us to prove the almost sure convergence of the occupation measure $\left(\mu_{t}\right)_{t \geq 0}$ toward $\alpha$. As in [2, 3, 21], we make use of stochastic approximation techniques (in the sense of $[2,5]$ ). These works strongly rely on the proof techniques of [16], which are based on technical regularity results that do not adapt well to the present setting. Our proof uses instead recent advances in the theory of quasi-stationary distributions [8] together with coupling arguments, in order to prove that the occupation measure dynamics are globally asymptotically stable. Combined with the general results on asymptotic pseudo trajectories of [2, 5], this entails the almost sure convergence of the occupation measure.

The paper is organised as follows. In Section 2, we state our main assumptions and results. In Section 3, we gather useful general results on quasi-stationary distributions from $[8,10]$ and prove new general results on a key operator $A$, which has its own interest and should be useful for future adaptation of the methods developed below. Section 4 is devoted to the proof of our main result, which consists in checking that the occupation measure of the resampling points is (up to a time change and linearization) an asymptotic pseudo-trajectory of a measure-valued dynamical system related to the operator $A$ (we refer the 
reader to [2] for an introduction to asymptotic pseudo-trajectories and their use in stochastic approximation theory).

\section{Main result}

From now on, we consider a diffusion process $\left(X_{t}, t \geq 0\right)$ in a connected bounded open set $D$ of $\mathbb{R}^{d}, d \geq 2$ with $C^{2}$ boundary $\partial D$ and absorbed at $\partial D$. We assume that $X$ is solution to the $\mathrm{SDE}$

$$
d X_{t}=\sigma\left(X_{t}\right) d B_{t}+b\left(X_{t}\right) d t
$$

where $\left(B_{t}, t \geq 0\right)$ is a $r$-dimensional Brownian motion, $b: D \rightarrow \mathbb{R}^{d}$ is bounded and continuous and $\sigma: D \rightarrow \mathbb{R}^{d \times r}$ is continuous, $\sigma \sigma^{*}$ is uniformly elliptic and for all $\rho>0$,

$$
\sup _{x, y \in D,|x-y|=\rho} \frac{|\sigma(x)-\sigma(y)|^{2}}{\rho} \leq g(\rho)
$$

for some function $g$ such that $\int_{0}^{1} g(r) d r<\infty$. Note that, in this case, the process $\left(Y_{t}, \mu_{t}\right)_{t \geq 0}$ described in the introduction is well-defined since one can prove that $\theta_{k} \rightarrow+\infty$ a.s. [4, Lemma 8.1].

In [7, Section 5.3], it was proved that, under the above regularity assumptions, the killed diffusion process $X$ admits a unique quasi-stationary distribution, i.e. a probability measure $\alpha$ on $D$ such that

$$
\alpha=\mathbb{P}_{\alpha}\left(X_{t} \in \cdot \mid t<\tau_{\partial}\right), \forall t \geq 0,
$$

where $\tau_{\partial}$ denotes the hitting time of $\partial D$ by the process. Moreover, it is well known that, in this case, there exists a positive constant $\lambda_{0}$ such that $\mathbb{P}_{\alpha}(t<$ $\left.\tau_{\partial}\right)=\exp \left(-\lambda_{0} t\right)$ for all $t \geq 0$ (see Section 3 for more results on quasi-stationary distributions).

Remark 1. In fact, the result of [7, Section 5.3] is stronger and entails the exponential convergence in total variation norm of the conditional law of $X$ toward $\alpha$, uniformly in the initial distribution. The proof relies on the fact that Conditions (A1) and (A2) as enunciated in the next section are satisfied by the process $X$ (see Section 3 for details and additional properties).

Remark 2. This last property was also proved to hold true for general one-dimensional diffusions in $D=[a,+\infty)$ or $D=[a, b]$ absorbed at the boundary of $D$ and coming down from infinity in [9] and for diffusion processes $X$ in compact, connected $C^{2}$ manifolds $M$ with $C^{2}$ boundary $\partial M$ absorbed at $\partial M$ when the infinitesimal generator of $X$ is given by $L=\frac{1}{2} \Delta+Z$, where $\Delta$ is the LaplaceBeltrami operator and $Z$ is a $C^{1}$ vector field in [7]. All the results of this paper, and in particular the next one, can be extended to these two situations.

The main result of this article is the following one. 
Theorem 2.1. For all bounded measurable function $f: D \rightarrow \mathbb{R}$, one has

$$
\mu_{t} f \underset{t \rightarrow+\infty}{\longrightarrow} \alpha f \quad \text { a.s. }
$$

Moreover, $\theta_{n} / n \rightarrow 1 / \lambda_{0}$ almost surely when $n \rightarrow+\infty$.

\section{Properties of the Green operator}

The results of Subsection 3 are valid for general absorbed Markov processes, not only for diffusion processes absorbed at the boundary of a domain. In Subsection 3.2, we provide properties on the measure-valued dynamical system induced by the Green operator of the process. Although not specific to diffusion processes, the later part uses the fact that the semi-group of the underlying process is Lipschitz regular.

\subsection{General properties}

Let us consider in this section a Markov process $\left(X_{t}, t \geq 0\right)$ on a measurable space $E \cup\{\partial\}$, absorbed in $\partial$ at time

$$
\tau_{\partial}:=\inf \left\{t \geq 0, X_{t}=\partial\right\},
$$

assumed a.s. finite. We also assume that $\mathbb{P}_{x}\left(t<\tau_{\partial}\right)>0$ for all $t \geq 0$ and all $x \in E$.

A probability measure $\alpha$ on $E$ is called a quasi-stationary distribution if

$$
\mathbb{P}_{\alpha}\left(X_{t} \in \cdot \mid t<\tau_{\partial}\right)=\alpha, \quad \forall t \geq 0
$$

It is well known that a probability measure $\alpha$ is a quasi-stationary distribution if and only if there exists a probability measure $\mu$ on $E$ such that

$$
\lim _{t \rightarrow+\infty} \mathbb{P}_{\mu}\left(X_{t} \in A \mid t<\tau_{\partial}\right)=\alpha(A)
$$

for all measurable subsets $A$ of $E$. The fact that $\alpha$ is a quasi-stationary distribution also implies the existence of a constant $\lambda_{0}>0$ such that

$$
\mathbb{P}_{\alpha}\left(t<\tau_{\partial}\right)=e^{-\lambda_{0} t} .
$$

In [8], the authors provide a necessary and sufficient condition on $X$ for the existence of a probability measure $\alpha$ on $E$ and constants $C, \gamma>0$ such that

$$
\left\|\mathbb{P}_{\mu}\left(X_{t} \in \cdot \mid t<\tau_{\partial}\right)-\alpha\right\|_{T V} \leq C e^{-\gamma t}, \quad \forall \mu \in \mathcal{M}_{1}(E), \quad t \geq 0,
$$

where $\mathcal{M}_{1}(E)$ is the set of probability measures on $E$ and $\|\cdot\|_{T V}$ is the total variation norm defined as $\left\|\mu_{1}-\mu_{2}\right\|_{T V}=\sup _{f \in \mathcal{B}_{b}(E),\|f\|_{\infty} \leq 1}\left|\mu_{1}(f)-\mu_{2}(f)\right|$ for all $\mu_{1}, \mu_{2} \in \mathcal{M}_{1}(E)$, where $\mathcal{B}_{b}(E)$ is the set of bounded measurable functions on $E$. This immediately implies that $\alpha$ is the unique quasi-stationary distribution of $X$ and that (3.1) holds for any initial probability measure $\mu$. 
The necessary and sufficient condition for (3.3) is given by the existence of a probability measure $\nu$ on $E$ and of constants $t_{0}, c_{1}, c_{2}>0$ such that

$$
\mathbb{P}_{x}\left(X_{t_{0}} \in \cdot \mid t_{0}<\tau_{\partial}\right) \geq c_{1} \nu, \quad \forall x \in E
$$

and

$$
\mathbb{P}_{\nu}\left(t<\tau_{\partial}\right) \geq c_{2} \mathbb{P}_{x}\left(t<\tau_{\partial}\right), \quad \forall t \geq 0, x \in E .
$$

Under Conditions (A1) and (A2), it follows from the general results of $[8$, Prop. 2.3] that there exists a bounded function $\eta: E \rightarrow[0, \infty)$ such that $\alpha(\eta)=$ 1 and, for all $x \in E$ and all $t \geq 0$,

$$
\left|e^{\lambda_{0} t} \mathbb{P}_{x}\left(t<\tau_{\partial}\right)-\eta(x)\right| \leq C^{\prime} e^{-\gamma t} .
$$

In the case of diffusion processes, $\eta$ is a nonnegative solution to $L \eta=-\lambda_{0} \eta$ where $L$ is the infinitesimal generator of the process $X$ in the set of bounded measurable functions equiped with the $L^{\infty}$ norm. The constant $\gamma$ is the same as in (3.3). In particular, there exists a constant $C^{\prime \prime}$ such that

$$
\mathbb{P}_{x}\left(t<\tau_{\partial}\right) \leq C^{\prime \prime} e^{-\lambda_{0} t}, \quad \forall t \geq 0, \forall x \in E .
$$

One can actually obtain a better bound combining Theorem 2.1 and Equation (3.2) of [10]: there exists a time $t_{1}>0$ and a constant $D$ such that, for all $t \geq t_{1}$ and all $x \in E$,

$$
\left|e^{\lambda_{0} t} \mathbb{P}_{x}\left(t<\tau_{\partial}\right)-\eta(x)\right| \leq D \eta(x) e^{-\gamma t} .
$$

We may - and will - assume without loss of generality that $D e^{-\gamma t_{1}} \leq 1 / 2$.

We denote by $P_{t}$ the (nonconservative) semigroup of the Markov process $\left(X_{t}, t \geq 0\right)$, acting on the set $\mathcal{B}_{b}(E)$ of bounded measurable functions on $E$ and defined for all such function $f$ by

$$
P_{t} f(x)=\mathbb{E}_{x}\left[f\left(X_{t}\right) 1_{t<\tau_{\partial}}\right], \quad \forall x \in E .
$$

Note that we made here the slight abuse of notation that $f(\partial) \cdot 0=0$. Because of (3.5), we can define the Green operator $A$ on $\mathcal{B}_{b}(E)$ as

$$
A f(x)=\mathbb{E}_{x}\left[\int_{0}^{\tau_{\partial}} f\left(X_{s}\right) d s\right]=\int_{0}^{\infty} P_{s} f(x) d s
$$

and this operator is bounded on $\mathcal{B}_{b}(E)$ equiped with the $L^{\infty}$ norm. Let $\mathcal{M}_{1}(E)$ be the set of probability measures on $E$. For all $\mu \in \mathcal{M}_{1}(E)$, we also define the notation

$$
\mu A f=\int_{E} A f(x) \mu(d x)=\mathbb{E}_{\mu}\left[\int_{0}^{\tau_{\partial}} f\left(X_{s}\right) d s\right]=\int_{0}^{\infty} \mu P_{s} f d s
$$

so that in particular $A f(x)=\delta_{x} A f$ and $\alpha A f=\int_{0}^{\infty} e^{-\lambda_{0} t} \alpha f d t=\alpha f / \lambda_{0}$. Since $A$ is bounded, the operator $e^{t A}$ is well-defined for all $t \geq 0$. 
Proposition 3.1. Assume that Conditions (A1) and (A2) are satisfied. Then, for all $\mu \in \mathcal{M}_{1}(E)$, all $f \in \mathcal{B}_{b}(E)$ and all $n \geq 1$, we have

$$
\left|\mu A^{n} f-\frac{\alpha(f) \mu(\eta)}{\lambda_{0}^{n}}\right| \leq \frac{\left(C C^{\prime \prime}+C^{\prime}\right)\|f\|_{\infty}}{\left(\lambda_{0}+\gamma\right)^{n}}
$$

where the constants $C, C^{\prime}, C^{\prime \prime}$ and $\gamma$ are those involved in (3.3), (3.4) and (3.5). We also have for some constant $B$

$$
\left\|\frac{\mu A^{n}}{\mu A^{n} 1}-\alpha\right\|_{T V} \leq \frac{B}{\mu(\eta)}\left(\frac{\lambda_{0}}{\lambda_{0}+\gamma}\right)^{n}
$$

and for all $t \geq 0$,

$$
\left\|\frac{\mu e^{t A}}{\mu e^{t A} 1}-\alpha\right\|_{T V} \leq \frac{B}{\mu(\eta)} e^{-t \frac{\gamma}{\lambda_{0}\left(\lambda_{0}+\gamma\right)}} .
$$

Proof. We first check by induction that for all $n \geq 1$,

$$
\mu A^{n} f=\int_{0}^{\infty} \frac{u^{n-1}}{(n-1) !} \mu P_{u} f d u .
$$

This is of course true for $n=1$. Assuming it is true for a given $n \geq 1$, we have

$$
\begin{aligned}
\mu A^{n+1} f & =\int_{0}^{\infty} \mu P_{s} A^{n} f d s \\
& =\int_{0}^{\infty} \int_{0}^{\infty} \frac{t^{n-1}}{(n-1) !} \mu P_{s} P_{t} f d t d s \\
& =\int_{0}^{\infty} \mu P_{u} f \int_{0}^{u} \frac{t^{n-1}}{(n-1) !} d t d u \\
& =\int_{0}^{\infty} \frac{u^{n}}{n !} \mu P_{u} f d u,
\end{aligned}
$$

which concludes the induction. Then, it follows from (3.3), (3.4) and (3.5) that

$$
\begin{aligned}
& \left|\mu A^{n} f-\int_{0}^{\infty} \frac{u^{n-1}}{(n-1) !} \alpha(f) e^{-\lambda_{0} u} \mu(\eta) d u\right| \\
& \quad \leq \int_{0}^{\infty} \frac{u^{n-1}}{(n-1) !}\left|\mu P_{u} f-\alpha(f) e^{-\lambda_{0} u} \mu(\eta)\right| d u \\
& \quad \leq \int_{0}^{\infty} \frac{u^{n-1}}{(n-1) !}\left[\mu P_{u} 1\left|\frac{\mu P_{u} f}{\mu P_{u} 1}-\alpha(f)\right|+\alpha(f)\left|\mu P_{u} 1-e^{-\lambda_{0} u} \mu(\eta)\right|\right] d u \\
& \quad \leq\left(C C^{\prime \prime}+C^{\prime}\right)\|f\|_{\infty} \int_{0}^{\infty} \frac{u^{n-1}}{(n-1) !} e^{-\left(\lambda_{0}+\gamma\right) u} d u .
\end{aligned}
$$

The inequality (3.8) follows. 
We then deduce from (3.8) that

$$
\begin{aligned}
\left\|\frac{\mu A^{n}}{\mu A^{n} 1}-\alpha\right\|_{T V} & \leq \frac{1}{\mu A^{n} 1}\left[\left\|\mu A^{n}-\mu(\eta) \lambda_{0}^{-n} \alpha\right\|_{T V}+\left|\mu A^{n} 1-\mu(\eta) \lambda_{0}^{-n}\right|\right] \\
& \leq \frac{2\left(C C^{\prime \prime}+C^{\prime}\right)}{\left(\lambda_{0}+\gamma\right)^{n} \mu A^{n} 1} .
\end{aligned}
$$

Now, it follows from (3.6) that

$$
\begin{aligned}
\mu A^{n} 1 & \geq \int_{t_{1}}^{\infty} \frac{u^{n-1}}{(n-1) !} \mu P_{u} 1 d u \\
& \geq \frac{\mu(\eta)}{2} \int_{t_{1}}^{\infty} \frac{u^{n-1}}{(n-1) !} e^{-\lambda_{0} u} d u \\
& =\frac{\mu(\eta) e^{-\lambda_{0} t_{1}}}{2}\left(\frac{t_{1}^{n-1}}{\lambda_{0}(n-1) !}+\frac{t_{1}^{n-2}}{\lambda_{0}^{2}(n-2) !}+\ldots+\frac{1}{\lambda_{0}^{n}}\right) \\
& \geq \frac{\mu(\eta) e^{-\lambda_{0} t_{1}}}{2 \lambda_{0}^{n}} .
\end{aligned}
$$

Combining the last two inequalities entails (3.9).

Similarly, for all $t \geq 0, f \in \mathcal{B}_{b}(E)$ and $\mu \in \mathcal{M}_{1}(E)$, we deduce from (3.8) that

$$
\begin{aligned}
\mid \frac{\mu e^{t A} f}{\mu e^{t A} 1} & -\alpha(f) \mid \\
& \leq \frac{1}{\mu e^{t A} 1} \sum_{n \geq 0} \frac{t^{n}}{n !}\left[\left|\mu A^{n} f-\mu(\eta) \lambda_{0}^{-n} \alpha(f)\right|+\alpha(f)\left|\mu(\eta) \lambda_{0}^{-n}-\mu A^{n} 1\right|\right] \\
& \leq \frac{2\left(C C^{\prime \prime}+C^{\prime}\right)\|f\|_{\infty}}{\mu e^{t A} 1} e^{\frac{t}{\lambda_{0}+\gamma}} .
\end{aligned}
$$

Now, it follows from (3.12) that

$$
\mu e^{t A} 1 \geq \frac{\mu(\eta) e^{-\lambda_{0} t_{1}}}{2} e^{\frac{t}{\lambda_{0}}} .
$$

The last two inequalities entail (3.10).

\subsection{Properties of a measure-valued dynamical system}

We begin with the following proposition, which ensures that $A$ is regularizing. In particular, for all $f \in C_{b}(D, \mathbb{R})$ (which denotes the set of bounded continuous functions from $D$ to $\mathbb{R}), x \in D \mapsto \delta_{x} A f$ is in $C_{b}(D, \mathbb{R})$. This Feller property implies that $\nu \mapsto \nu A$ is continuous with respect to the weak topology on the set $\mathcal{M}(D)$ of non-negative measures with finite mass on $D$. Similarly, one deduces that $(t, \nu) \in[0,+\infty) \times \mathcal{M}(D) \mapsto \nu e^{t A} \in \mathcal{M}(D)$ is continuous. 
Proposition 3.2. For all bounded measurable functions $f: D \rightarrow \mathbb{R}$, the application $x \mapsto \delta_{x} A f$ is Lipschitz continuous, with Lipschitz norm proportional to $\|f\|_{\infty}$.

Proof. From Priola and Wang [17], one deduces that there exists a constant $C_{\text {Lip }}>0$ which does not depend on $f$ such that, for all $t>0$ and all $x, y \in D$,

$$
\left|\delta_{x} P_{t} f-\delta_{y} P_{t} f\right| \leq \frac{C_{L i p}}{1 \wedge \sqrt{t}}\|f\|_{\infty} .
$$

Applying this inequality to $x \mapsto \delta_{x} P_{t} f$ at time 1 and using inequality (3.5), one deduces that

$$
\left|\delta_{x} P_{t+1} f-\delta_{y} P_{t+1} f\right| \leq C_{L i p}\left\|P_{t} f\right\|_{\infty} \leq C_{L i p} C^{\prime \prime}\|f\|_{\infty} e^{-\lambda_{0} t} .
$$

As a consequence,

$\left|\delta_{x} A f-\delta_{y} A f\right| \leq|x-y| \int_{0}^{1} \frac{C_{L i p}}{\sqrt{t}}\|f\|_{\infty} d t+|x-y| \int_{1}^{\infty} C_{L i p} C^{\prime \prime}\|f\|_{\infty} e^{-\lambda_{0}(t-1)} d t$,

which concludes the proof of Proposition 3.2.

The following proposition states the uniqueness of the evolution equation satisfied by the continuous process $\left(\nu e^{t A} / \nu e^{t A} 1_{D}\right)_{t \geq 0}$.

Proposition 3.3. For each probability measure $\nu$ on $D$, the equation

$$
\frac{d \varphi_{t}}{d t}=F\left(\varphi_{t}\right), \quad \varphi_{0}=\nu,
$$

where $F$ is a measure valued function defined, for all non-negative finite measures $\nu$ on $D$ by

$$
F(\nu)=\nu A-\left(\nu A 1_{D}\right) \nu,
$$

admits a unique weak solution in $C([0,+\infty), \mathcal{M}(D))$, where $\mathcal{M}(D)$ is equiped with the weak topology, in the sense that, for all bounded continuous function $f: D \rightarrow \mathbb{R}$ and all $t \geq 0$,

$$
\varphi_{t}(f)=\varphi_{0}(f)+\int_{0}^{t} F\left(\varphi_{s}\right)(f) d s .
$$

In addition, this unique weak solution takes its values in $\mathcal{M}_{1}(D)$ and is given by $\varphi_{t}=\nu e^{t A} / \nu e^{t A} 1_{D}$.

Proof. The fact that $\left(\nu e^{t A} / \nu e^{t A} 1_{D}\right)_{t \geq 0}$ satisfies (3.13) is immediate. Let us check that this equation has no other solution. In order to do so, we consider one of its solutions $\left(\varphi_{t}\right)_{t \geq 0}$ and introduce the measure valued process defined by

$$
\tilde{\varphi}_{t}=\exp \left(\int_{0}^{t} \varphi_{s}\left(A 1_{D}\right) d s\right) \varphi_{t}, \quad \forall t \geq 0 .
$$


This process is weak solution to the linear evolution equation

$$
\frac{\partial \tilde{\varphi}_{t}}{\partial t}=\tilde{\varphi}_{t} A, \tilde{\varphi}_{0}=\nu
$$

whose unique weak solution is $t \mapsto \nu e^{t A}$. Indeed, let $t \mapsto \mu_{t}, \nu_{t}$ be two weak solutions to the linear equation. Set $\left|\mu_{t}-\nu_{t}\right|=\sup _{f}\left|\mu_{t} f-\nu_{t} f\right|$ where the supremum is taken over the set of continuous function $f: D \mapsto \mathbb{R}$ such that $\|f\|_{\infty} \leq 1$. Then $t \mapsto\left|\mu_{t}-\nu_{t}\right|$ is lower semicontinuous, hence measurable, as a supremum of continuous functions. Thus, by Gronwall's lemma (measurable version, see [13]), $\left|\mu_{t}-\nu_{t}\right| \leq\left|\mu_{0}-\nu_{0}\right| e^{\|A\| t}$. This proves uniqueness.

As a consequence, for all $t \geq 0$,

$$
\varphi_{t}=\frac{\tilde{\varphi}_{t}}{\tilde{\varphi}_{t} 1_{D}}=\frac{\nu e^{t A}}{\nu e^{t A} 1_{D}},
$$

which concludes the proof of Proposition 3.3.

\section{Proof of Theorem 2.1}

The general idea of the proof is inspired from [2] and consists in proving that a time-change of the sequence of probability measures

$$
\eta_{n}=\frac{1}{n} \sum_{i=1}^{n} \delta_{Z_{i}}, \quad \text { where } \quad Z_{i}:=Y_{\theta_{i}}
$$

is an asymptotic pseudo-trajectory (see [2] for the definition of an asymptotic pseudo-trajectory) of a measure-valued dynamical system related to the nor-

malized semigroup $\frac{\nu e^{t A}}{\nu e^{t A} 1_{D}}$. The asymptotic properties given in Proposition 3.1 then allow to deduce that $\eta_{n}$ almost surely converges to $\alpha$. The proof is divided in three steps. First, we prove in Subsection 4.1 tightness properties on the measure-valued process $\left(\mu_{t}\right)_{t \geq 0}$. The convergence of $\eta_{n}$ to $\alpha$ is proved in Subsection 4.2, using a key lemma on asymptotic pseudo-trajectories properties for $\eta_{n}$, proved in Subsection 4.3. Theorem 2.1 is then be deduced from the convergence of $\eta_{n}$ using martingale arguments in Subsection 4.4.

\subsection{Tightness}

The following proposition entails that the paths of $\left(\mu_{t}, t \geq 0\right)$ are a.s. relatively compact in the set of probability measures on $D$ endowed with the weak topology.

Proposition 4.1. For all $\varepsilon>0$, there exists $\eta>0$ such that, almost surely,

$$
\liminf _{t \rightarrow+\infty} \mu_{t}(\{x \in D: d(x, \partial D)<\eta\}) \leq \varepsilon
$$


Proof. Let $\phi_{D}: D \rightarrow \mathbb{R}_{+}$be the distance to $\partial D$. There exists a neighborhood $\mathcal{N}$ of $\partial D$ in $D$ where $\phi_{D}$ is $C_{b}^{2}$ so that we can apply Itô's formula: for all $t \geq 0$ such that $Y_{t} \in \mathcal{N}$,

$d \phi_{D}\left(Y_{t}\right)=\left(\sigma\left(Y_{t}\right)^{*} \nabla \phi_{D}\left(Y_{t}\right)\right) \cdot d B_{t}+\nabla \phi_{D}\left(Y_{t}\right) \cdot b\left(Y_{t}\right) d t+\frac{1}{2} \operatorname{Tr}\left(\sigma\left(Y_{t}\right)^{*} D^{2} \phi_{D}\left(Y_{t}\right) \sigma\left(Y_{t}\right)\right) d t$.

We introduce the random time-change $\tau(t)$ such that

$$
\int_{0}^{\tau(t)}\left(1_{Y_{s} \in \mathcal{N}}\left\|\sigma\left(Y_{s}\right)^{*} \nabla \phi_{D}\left(Y_{s}\right)\right\|_{2}^{2}+1_{Y_{s} \notin \mathcal{N}}\right) d s=t
$$

and we observe that there exist constants $0<c_{0}<C_{0}<\infty$ such that $c_{0} \leq$ $\tau^{\prime}(t) \leq C_{0}$ for all $t \geq 0$. Then, there exists a Brownian motion $W$ such that the process $Z_{t}:=\phi_{D}\left(Y_{\tau(t)}\right)$ satisfies

$$
d Z_{t}=d W_{t}+H_{t} d t, \quad \forall t \text { s.t. } Z_{t} \in \mathcal{N},
$$

where the process $H$ is progressively measurable and bounded by a constant $\bar{H}>0$.

We introduce $a>0$ such that $\{x \in D: d(x, \partial D) \leq 2 a\} \subset \mathcal{N}$ and the reflected drifted Brownian motion $\left(\bar{Z}_{t}, t \geq 0\right)$ solution to

$$
d \bar{Z}_{t}=d W_{t}-\bar{H} d t+d L_{t}^{0}-d L_{t}^{a}, \quad \forall t \geq 0
$$

and such that $\bar{Z}_{0}=\phi_{D}\left(Y_{0}\right) \wedge a$, where $L_{t}^{x}$ is the local time of $\bar{Z}$ at $x$ at time $t$.

Since the jumps of $Z$ are positive, one can prove following [20, Prop. 2.2] that $\bar{Z}_{t} \leq Z_{t}$ a.s. for all $t \geq 0$. Moreover, the process $\bar{Z}$ is ergodic and satisfies almost surely

$$
\frac{1}{t} \int_{0}^{t} \delta_{\bar{Z}_{s}} d s \underset{t \rightarrow+\infty}{\longrightarrow} m
$$

where $m(d x)=C e^{-2 \bar{H} x} 1_{[0, a]}(x) d x$ is the stationary distribution of $\bar{Z}$ on $[0, a]$.

Now, for all $\varepsilon>0$, there exists $\eta>0$ such that $m(0, \eta)<\varepsilon$. Hence, almost surely for all $t$ large enough

$$
\begin{aligned}
\mu_{\tau(t)}(\{x \in D: d(x, \partial D)<\eta\}) & \leq \frac{1}{\tau(t)} \int_{0}^{\tau(t)} 1_{\bar{Z}_{\tau^{-1}(s)}<\eta} d s \leq \frac{1}{\tau(t)} \int_{0}^{t} 1_{\bar{Z}_{u}<\eta} \tau^{\prime}(u) d u \\
& \leq \frac{C_{0}}{c_{0} t} \int_{0}^{t} 1_{\bar{Z}_{u}<\eta} d u \leq \frac{C_{0} \varepsilon}{c_{0}} .
\end{aligned}
$$

Since $\tau: \mathbb{R}_{+} \rightarrow \mathbb{R}_{+}$is continuous and $\tau^{\prime}(t) \geq c_{0}$ for all $t \geq 0$, this concludes the proof of Proposition 4.1.

The previous proposition entails that, for all $\varepsilon>0$, there exists $\eta>0$ such that, almost surely, $\theta_{n} \mu_{\theta_{n}}(\{x \in D: d(x, \partial D) \geq \eta\}) \geq(1-2 \varepsilon) \theta_{n}$ for $n$ large enough. The following proposition is of a slightly different nature and it will be used later in order to prove that there exists a constant $c>0$ such that, almost surely, $\theta_{n} \mu_{\theta_{n}}(\{x \in D: d(x, \partial D) \geq \eta\}) \geq c n$ for $n$ large enough. 
Proposition 4.2. For all $\varepsilon>0$, there exists $\eta>0$ such that, almost surely, one has

$$
\liminf _{n \rightarrow+\infty} \frac{1}{n} \sum_{i=1}^{n} 1_{d\left(Y_{\theta_{i}}, \partial D\right) \geq \eta} \geq 1-2 \varepsilon
$$

and

$$
\liminf _{n \rightarrow+\infty} \frac{\theta_{n}}{n} \geq(1-2 \varepsilon) c_{0} \mathbb{E}\left(\bar{T}_{0}\right),
$$

where $\bar{T}_{0}=\inf \left\{t \geq 0, \bar{Z}_{t}=0\right\}, \bar{Z}_{0}=\eta$ and the constant $c_{0}$ and the process $\bar{Z}$ were defined in the proof of Proposition 4.1.

Proof. Fix $\varepsilon>0$. From Proposition 4.1, there exists $\eta>0$ such that, almost surely, $\mu_{\theta_{n}}(\{x \in D: d(x, \partial D) \geq \eta\}) \geq 1-2 \varepsilon$ for $n$ large enough. For all $k \in \mathbb{N}=\{1,2, \ldots\}$, we define the random variable in $\mathbb{N} \cup\{+\infty\}$

$$
v_{k}=\inf \left\{n \geq k, \mu_{\theta_{n}}(\{x \in D: d(x, \partial D) \geq \eta\})<1-2 \varepsilon\right\},
$$

so that $\mathbb{P}\left(\cup_{k=1}^{\infty}\left\{v_{k}=+\infty\right\}\right)=1$. We also define the sequence of points $\left(Z_{n}^{k}\right)_{n \geq 0}$ in $D$ by

$$
Z_{n}^{k}= \begin{cases}Y_{\theta_{n}} & \text { if } n<v_{k} \\ x_{0} & \text { if } n \geq v_{k}\end{cases}
$$

where $x_{0}$ is an arbirary point in $\{x \in D: d(x, \partial D) \geq \eta\}$. By definition, the law of $Y_{\theta_{n}}=X_{0}^{(n+1)}$ conditionally to $\mu_{\theta_{1}}, \ldots, \mu_{\theta_{n}}$ and $Y_{\theta_{0}}, Y_{\theta_{1}}, \ldots, Y_{\theta_{n-1}}$ is $\mu_{\theta_{n}}$. Moreover, $\left\{n<v_{k}\right\}$ is measurable with respect to $\mu_{\theta_{1}}, \ldots, \mu_{\theta_{n}}$, and hence, for all $n \geq k$, (we denote by $\mathbb{P}^{n}$ the probability conditionally to $\mu_{\theta_{1}}, \ldots, \mu_{\theta_{n}}$ and $\left.Y_{\theta_{1}}, \ldots, Y_{\theta_{n-1}}\right)$

$$
\begin{aligned}
\mathbb{P}^{n}\left(d\left(Z_{n}^{k}, \partial D\right) \geq \eta\right) & =\mathbb{P}^{n}\left(d\left(Y_{\theta_{n}}, \partial D\right) \geq \eta\right) 1_{n<v_{k}}+1_{n \leq v_{k}} \\
& \geq \mu_{\theta_{n}}(\{x \in D: d(x, \partial D) \geq \eta\}) 1_{n<v_{k}}+1_{n \leq v_{k}} \geq 1-2 \varepsilon
\end{aligned}
$$

Using the law of large numbers for submartingales, this implies that, almost surely and for all $k \geq 1$,

$$
\liminf _{n \rightarrow+\infty} \frac{1}{n} \sum_{i=1}^{n} 1_{d\left(Z_{i}^{k}, \partial D\right) \geq \eta} \geq 1-2 \varepsilon .
$$

Observing that, almost surely, there exists $k \geq 1$ such that $Z_{n}^{k}=Y_{\theta_{n}}$ for all $n \geq 1$, this concludes the proof of (4.2).

To prove (4.3), we observe that, due to the coupling argument of the proof of Proposition 4.1,

$$
\theta_{n} \geq \sum_{i=1}^{n} 1_{d\left(Z_{i}^{k}, \partial D\right) \geq \eta} \bar{T}_{0}^{(i)}
$$

where $\left(\bar{T}_{0}^{(i)}\right)_{i \geq 1}$ are i.i.d. copies of $\bar{T}_{0}$ such that $\bar{T}_{0}^{(i)}$ is independent of $Z_{1}^{k}, \ldots, Z_{i}^{k}$ for all $i \geq 1$. Therefore, we can use the law of large numbers for submartingales as above to conclude the proof of Proposition 4.2. 


\subsection{Study of the empirical measure of the resampling points}

In this subsection, we focus on the behaviour of the random sequence of measures $\left(\eta_{n}\right)_{n \geq 1}$ defined in (4.1). Our aim is to prove the following proposition using the theory of pseudo-asymptotic trajectories.

Proposition 4.3. The sequence of probability measures $\left(\eta_{n}\right)_{n \in \mathbb{N}}$ converges almost surely to $\alpha$ with respect to the weak topology.

Proof. We follow an approach inspired from [2]. Let $\left(\tau_{n}\right)_{n \geq 1}$ be defined as $\tau_{1}=0$ and $\tau_{n}=\gamma_{2}+\gamma_{3}+\cdots+\gamma_{n}$ for $n \geq 2$, where

$$
\gamma_{n+1}=\frac{1}{(n+1) \eta_{n} A 1_{D}}, \quad \forall n \geq 1 .
$$

We consider the linearly interpolated version $\left(\widetilde{\eta}_{t}\right)_{t \in[1,+\infty)}$ of $\left(\eta_{n}\right)_{n \in \mathbb{N}}$ defined, for all $n \geq 0$ and all $t \in\left[\tau_{n}, \tau_{n+1}\right]$, by

$$
\widetilde{\eta}_{t}=\eta_{n}+\frac{t-\tau_{n}}{\tau_{n+1}-\tau_{n}}\left(\eta_{n+1}-\eta_{n}\right)
$$

where we define by convention $\eta_{0}=\delta_{x_{0}}$ for some fixed $x_{0} \in D$.

Let $\left(f_{k}\right)_{k \in \mathbb{N}}$ be a sequence of bounded continuous functions from $D$ to $\mathbb{R}$ such that the metric

$$
d\left(\nu_{1}, \nu_{2}\right)=\sum_{k=0}^{\infty} \frac{\left|\nu_{1} f_{k}-\nu_{2} f_{k}\right|}{2^{k}\left\|f_{k}\right\|_{\infty}}
$$

metrizes the weak topology on measures on $D$.

The main point of the proof consists in using [2, Theorem 3.2] to prove that $\widetilde{\eta}$ is an asymptotic pseudo-trajectory of (3.13). By Proposition 3.3, this means in our setting that, for all $T>0$,

$$
\lim _{t \rightarrow+\infty} \sup _{s \in[0, T]} d\left(\widetilde{\eta}_{t+s}, \frac{\widetilde{\eta}_{t} e^{s A}}{\widetilde{\eta}_{t} e^{s A} 1_{D}}\right)=0 .
$$

This is stated in the next lemma, proved in the next subsection.

Lemma 4.4. The measure-valued process $\widetilde{\eta}$ is almost surely an asymptotic pseudo-trajectory for the distance $d$ on the set of probability measures on $D$ of the semi-flow induced by (3.13) and defined in Proposition 3.3.

Once this is proved, Proposition 4.3 follows easily: indeed $\left(\widetilde{\eta}_{t}\right)_{t \geq 0}$ is almost surely a relatively compact asymptotic pseudo-trajectory of the semi-flow induced by (3.13) for which $\{\alpha\}$ is a compact global attractor, which implies the result (see for instance [4, Corollary 5.3] and [2, 5]). 


\subsection{Proof of Lemma 4.4}

For all $n \geq 1$, we have

$$
\eta_{n+1}-\eta_{n}=\frac{\delta_{Z_{n+1}}-\eta_{n}}{n+1}=\gamma_{n+1}\left(F\left(\eta_{n}\right)+U_{n+1}\right),
$$

where, recalling the definition of $A$ in (3.7) and of $F$ in (3.14),

$$
\gamma_{n+1}=\frac{1}{(n+1) \eta_{n} A 1_{D}} \quad \text { and } \quad U_{n+1}=\left(\eta_{n} A 1_{D}\right) \delta_{Z_{n+1}}-\eta_{n} A .
$$

Fix $\varepsilon \in(0,1 / 4)$ and $\eta>0$ small enough so that the conclusions of Proposition 4.2 hold true. Setting $c:=\inf _{x \in D, d(x, \partial D)>\eta} \frac{\delta_{x} A 1_{D}}{2} \wedge \frac{c_{0} \mathbb{E} \bar{T}_{0}}{2}$, which is positive by Proposition 3.2, we define for all $k \geq 1$ the random variable in $\mathbb{N} \cup\{+\infty\}$

$$
\sigma_{k}=\inf \left\{n \geq k, \eta_{n} A 1_{D} \leq c \text { or } \theta_{n} \leq c n\right\} .
$$

The conclusion of Proposition 4.2 entails that $\mathbb{P}\left(\cup_{k=1}^{\infty}\left\{\sigma_{k}=+\infty\right\}\right)=1$.

Following [2], before proving Lemma 4.4, we begin by proving the next lemma.

Lemma 4.5. Almost surely, for all bounded measurable function $f: D \rightarrow \mathbb{R}$, the numeric sequence $\left(\sum_{\ell=1}^{n} \gamma_{\ell} U_{\ell} f\right)_{n}$ admits a finite limit when $n \rightarrow+\infty$.

Proof. For all $\ell \geq 0$, we introduce $\mathcal{G}_{\ell}$ the $\sigma$-field generated by $\mu_{\theta_{1}}, \ldots, \mu_{\theta_{\ell+1}}$, $\theta_{1}, \ldots, \theta_{\ell+1}$ and $Z_{1}, \ldots, Z_{\ell}$. Fix $k \geq 1$. We start by observing that

$$
\left\{\ell \leq \sigma_{k}\right\}=\left\{\forall n \in\{k, k+1, \ldots, \ell-1\}, \eta_{n} A 1_{D}>c \text { and } \theta_{n}>c n\right\} \in \mathcal{G}_{\ell-1},
$$

so that $\sigma_{k}$ is predictable with respect to the filtration $\left(\mathcal{G}_{\ell}\right)_{\ell \geq 0}$.

Following [18, Lemma 1], we define $N_{\ell}=\gamma_{\ell} U_{\ell} f$ and

$$
M_{n}^{(k)}=\sum_{\ell=1}^{n \wedge \sigma_{k}}\left(N_{\ell}-\mathbb{E}_{\ell-1} N_{\ell}\right)
$$

where $\mathbb{E}_{\ell-1}$ denotes the expectation conditionally to $\mathcal{G}_{\ell-1}$. Observe $M_{n}^{(k)}$ is a martingale with respect to $\left(\mathcal{G}_{\ell}\right)_{\ell \geq 0}$ and that

$$
N_{\ell}=\frac{1}{\ell}\left(f\left(Z_{\ell}\right)-\frac{\eta_{\ell-1} A f}{\eta_{\ell-1} A 1_{D}}\right) \quad \text { and } \quad \mathbb{E}_{\ell-1} N_{\ell}=\frac{1}{\ell}\left(\mu_{\theta_{\ell}} f-\frac{\eta_{\ell-1} A f}{\eta_{\ell-1} A 1_{D}}\right) .
$$

We have, for all $n \geq 0$

$$
\begin{aligned}
\mathbb{E}\left|M_{n}^{(k)}\right|^{2} & =\sum_{\ell=1}^{n} \mathbb{E}\left[\left|N_{\ell}-\mathbb{E}_{\ell-1} N_{\ell}\right|^{2} 1_{\ell \leq \sigma_{k}}\right] \\
& \leq 2 \sum_{\ell=1}^{n} \mathbb{E}\left[\left|N_{\ell}\right|^{2}+\left|\mathbb{E}_{\ell-1} N_{\ell}\right|^{2}\right] \leq 4 \sum_{\ell=1}^{n} \frac{\|f\|_{\infty}^{2}}{\ell^{2}}
\end{aligned}
$$


As a consequence, the martingale $\left(M_{n}^{(k)}\right)_{n>0}$ is uniformly bounded in $L^{2}$ and hence converges almost surely. Let us now prove that $\sum_{\ell=1}^{n \wedge \sigma_{k}} \mathbb{E}_{\ell-1} N_{\ell}$ converges almost surely when $n \rightarrow+\infty$.

We have, for all $\ell \geq 1$,

$$
\mathbb{E}\left|\mathbb{E}_{\ell-1}\left[N_{\ell}\right] 1_{\ell \leq \sigma_{k}}\right|=\frac{1}{\ell} \mathbb{E}\left|\mu_{\theta_{\ell}} f 1_{\ell \leq \sigma_{k}}-\frac{\eta_{\ell-1} A f}{\eta_{\ell-1} A 1_{D}} 1_{\ell \leq \sigma_{k}}\right| .
$$

For all $\ell<k$, this quantity is almost surely bounded by $2\|f\|_{\infty} / \ell$. For all $\ell \geq k$, the definition of $\sigma_{k}$ entails that

$$
\begin{aligned}
\mathbb{E}\left|\mathbb{E}_{\ell-1}\left[N_{\ell}\right] 1_{\ell \leq \sigma_{k}}\right| \leq & \frac{1}{\ell} \mathbb{E}\left|\left(\frac{1}{\theta_{\ell}}-\frac{1}{(\ell-1) \eta_{\ell-1} A 1_{D}}\right) \theta_{\ell} \mu_{\theta_{\ell}} f 1_{\ell \leq \sigma_{k}}\right| \\
& +\frac{1}{c \ell(\ell-1)} \mathbb{E}\left[\left|\theta_{\ell} \mu_{\theta_{\ell}} f-(\ell-1) \eta_{\ell-1} A f\right| 1_{\ell \leq \sigma_{k}}\right] .
\end{aligned}
$$

We first consider the term in (4.6). It follows from the fact that $\left(\theta_{\ell+1} \mu_{\theta_{\ell+1}} f-\right.$ $\left.\ell \eta_{\ell} A f\right)_{\ell \geq 0}$ is a $\left(\mathcal{G}_{\ell}\right)_{\ell \geq 0}$-martingale and from Cauchy-Schwarz inequality that

$$
\begin{aligned}
\mathbb{E}\left[\left|\theta_{\ell} \mu_{\theta_{\ell}} f-(\ell-1) \eta_{\ell-1} A f\right| 1_{\ell \leq \sigma_{k}}\right]^{2} \\
\leq \mathbb{E}\left[\left|\theta_{\ell} \mu_{\theta_{\ell}} f-(\ell-1) \eta_{\ell-1} A f\right|^{2}\right] \\
=\sum_{i=1}^{\ell} \mathbb{E}\left[\left|\int_{0}^{\tau_{\partial}^{(i)}} f\left(X_{s}^{(i)}\right) d s-\delta_{Z_{i-1}} A f\right|^{2}\right] \\
\leq 2\|f\|_{\infty}^{2} \sum_{i=1}^{\ell} \mathbb{E}\left(\left(\tau_{\partial}^{(i)}\right)^{2}\right) \leq 2\|f\|_{\infty}^{2} \sup _{x \in D} \mathbb{E}_{x}\left(\tau_{\partial}^{2}\right),
\end{aligned}
$$

where $\sup _{x \in D} \mathbb{E}_{x}\left(\tau_{\partial}^{2}\right)<+\infty$ since, by (3.5),

$\mathbb{E}_{x}\left(\tau_{\partial}^{2}\right)=2 \mathbb{E}_{x}\left(\int_{0}^{+\infty} t 1_{t<\tau_{\partial}} d t\right) \leq 2 \int_{0}^{+\infty} t \mathbb{P}_{x}\left(t<\tau_{\partial}\right) d t \leq 2 \int_{0}^{+\infty} t C^{\prime \prime} e^{-\lambda_{0} t} d t$

Consider now the term in (4.5).

$$
\begin{aligned}
\mathbb{E} \mid\left(\frac{1}{\theta_{\ell}}-\frac{1}{(\ell-1) \eta_{\ell-1} A 1_{D}}\right) & \left.\theta_{\ell} \mu_{\theta_{\ell}} f 1_{\ell \leq \sigma_{k}}\right|^{2} \\
& \leq\|f\|_{\infty}^{2} \mathbb{E}\left|\left(1-\frac{\theta_{\ell}}{(\ell-1) \eta_{\ell-1} A 1_{D}}\right)^{2} 1_{\ell \leq \sigma_{k}}\right| \\
& \leq \frac{\|f\|_{\infty}^{2}}{c^{2}(\ell-1)^{2}} \mathbb{E}\left|\left((\ell-1) \eta_{\ell-1} A 1_{D}-\theta_{\ell}\right)^{2} 1_{\ell \leq \sigma_{k}}\right| \\
& \leq \frac{\|f\|_{\infty}^{2}}{c^{2}(\ell-1)^{2}} 2 \ell \sup _{x \in D} \mathbb{E}_{x}\left(\tau_{\partial}^{2}\right),
\end{aligned}
$$


where we used (4.7) with $f=1_{D}$ to obtain the last inequality.

We deduce that $\mathbb{E}\left|\mathbb{E}_{\ell-1}\left(N_{\ell} 1_{\ell \leq \sigma_{k}}\right)\right|$ is $\mathcal{O}\left(\ell^{-3 / 2}\right)$ (beware that the $\mathcal{O}$ may depend on $k$ ), so that $\mathbb{E}\left|\sum_{\ell=1}^{n \wedge \sigma_{k}} \mathbb{E}_{\ell-1} N_{\ell}\right|<+\infty$ and hence that $\sum_{\ell=1}^{n \wedge \sigma_{k}} \mathbb{E}_{\ell-1} N_{\ell}<$ $\infty$ almost surely.

Because of the a.s. convergence of the sequence $\left(M_{n}^{(k)}\right)_{n \in \mathbb{N}}$, we conclude that $\left(\sum_{\ell=1}^{n \wedge \sigma_{k}} N_{\ell}\right)_{n \in \mathbb{N}}$ converges almost surely when $n \rightarrow+\infty$ for all $k \geq 1$. Since, almost surely, there exists $k \geq 1$ such that $\sigma_{k}=+\infty$, this concludes the proof of Lemma 4.5.

Proof of Lemma 4.4. We introduce the time-changed version $\left(\bar{\eta}_{t}\right)_{t \in[1,+\infty)}$ of the sequence $\left(\eta_{n}\right)_{n \in \mathbb{N}}$ as $\bar{\eta}_{t}=\eta_{n}$ for all $n \geq 1$ and all $t \in\left[\tau_{n}, \tau_{n+1}\right)$. We also define $\bar{U}_{t}=U_{n+1}$ for all $t \in\left[\tau_{n}, \tau_{n+1}\right)$.

To apply [2, Theorem 3.2], one needs to prove that $\left(\widetilde{\eta}_{t}\right)_{t \geq 0}$ is almost surely relatively compact, that it is almost surely uniformly continuous and that all limit points of $\left(\Theta_{t}(\widetilde{\eta})\right)_{t \geq 0}$ in $C\left(\mathbb{R}_{+}, \mathcal{M}(D)\right)$, endowed with the topology of uniform convergence for the metric $d$ on compact time inervals, are almost surely weak solutions of $(3.13)$, where $\Theta_{t}(\widetilde{\eta}):=\left(\widetilde{\eta}_{t+s}\right)_{s \geq 0}$.

The fact that $\left(\widetilde{\eta}_{t}\right)_{t \geq 0}$ is relatively compact is an immediate consequence of Proposition 4.2 and the almost surely uniform continuity is also immediately obtained from the construction of $\widetilde{\eta}$, since for all $s, t \in\left[\tau_{n}, \tau_{n+1}\right]$,

$$
\begin{aligned}
d\left(\widetilde{\eta}_{s}, \widetilde{\eta}_{t}\right) & \leq \sum_{k=0}^{\infty} \frac{|s-t|}{2^{k} \gamma_{n+1}\left\|f_{k}\right\|_{\infty}}\left|\frac{f_{k}\left(Z_{n+1}\right)}{n+1}-\frac{f_{k}\left(Z_{1}\right)+\ldots+f_{k}\left(Z_{n}\right)}{n(n+1)}\right| \\
& \leq \frac{4}{(n+1) \gamma_{n+1}}|s-t|
\end{aligned}
$$

and since $\inf _{n \geq 1}(n+1) \gamma_{n+1}>0$ almost surely by Proposition 4.2 .

In order to prove the last point, we adapt the method developed in [2, Proposition 4.1]. Assume that there exists an increasing sequence of positive numbers $\left(t_{n}\right)_{n \geq 0}$ converging to $+\infty$ such that $\left(\Theta_{t_{n}}(\widetilde{\eta})\right)_{n \geq 0}$ converges to an element $\widetilde{\eta}^{\infty}$ in $C\left(\mathbb{R}_{+}, \mathcal{M}(D)\right)$ with respect to the uniform convergence on compact time intervals. Our aim is to prove that $\widetilde{\eta}^{\infty}$ is a weak solution to (3.13).

For all $f \in C_{b}\left(D, \mathbb{R}_{+}\right)$, define $L_{F}^{f}: C\left(\mathbb{R}_{+}, \mathcal{M}(D)\right) \rightarrow \mathbb{R}^{[0,+\infty)}$ by

$$
L_{F}^{f}(\nu)(t)=\nu_{0} f+\int_{0}^{t} F\left(\nu_{s}\right) f d s, \quad \forall \nu \in C\left(\mathbb{R}_{+}, \mathcal{M}(D)\right),
$$

so that, using the equality $\int_{t}^{t+s}\left(F\left(\bar{\eta}_{u}\right)+\bar{U}_{u}\right) d u=-\widetilde{\eta}_{t}+\widetilde{\eta}_{t+s}$,

$$
\Theta_{t}(\widetilde{\eta}) f=L_{F}^{f}\left(\Theta_{t}(\widetilde{\eta})\right)+A_{t}^{f}+B_{t}^{f}
$$

where, for all $s \geq 0$,

$$
A_{t}^{f}(s)=\int_{t}^{t+s}\left(F\left(\bar{\eta}_{u}\right) f-F\left(\widetilde{\eta}_{u}\right) f\right) d u \quad \text { and } \quad B_{t}^{f}(s)=\int_{t}^{t+s} \bar{U}_{u} f d u .
$$


For all $u \in[0,+\infty)$, let us denote by $n_{u}$ the unique non-negative integer such that $u \in\left[\tau_{n_{u}}, \tau_{n_{u}+1}\right)$. Then, proceeding as in (4.8), one easily checks that

$$
\left|\bar{\eta}_{u} g-\widetilde{\eta}_{u} g\right| \leq \frac{2\|g\|_{\infty}}{n_{u}+1}, \quad \forall g \in C_{b}\left(D, \mathbb{R}_{+}\right) .
$$

Since $n_{u} \rightarrow+\infty$ when $u \rightarrow+\infty$ and since $F(\nu) f=\nu A f-\left(\nu A 1_{D}\right) \nu f$, where $A f$ and $A 1_{D}$ are bounded continuous functions, we deduce that $A_{t}^{f}(s)$ converges to 0 when $t \rightarrow \infty$.

Also, for all $t \in\left[\tau_{n}, \tau_{n+1}\right)$ and $t+s \in\left[\tau_{n+m}, \tau_{n+m+1}\right)$,

$$
\begin{aligned}
\left|B_{t}^{f}(s)\right| & \leq\left(\tau_{n+1}-t\right)\left|U_{n+1} f\right|+\left|\sum_{\ell=n+1}^{n+m-1} \gamma_{\ell+1} U_{\ell+1} f\right|+\left(s-\tau_{n+m}\right)\left|U_{n+m+1} f\right| \\
& \leq \gamma_{n+1}\left|U_{n+1} f\right|+\left|\sum_{\ell=n+1}^{n+m-1} \gamma_{\ell+1} U_{\ell+1} f\right|+\gamma_{n+m+1}\left|U_{n+m+1} f\right| .
\end{aligned}
$$

Hence Lemma 4.5 implies that $B_{t}^{f}(s)$ also goes to 0 when $t \rightarrow+\infty$.

Finally, since $L_{F}^{f}$ is clearly sequentially continuous in $C([0,+\infty), \mathcal{M}(D))$, one finally deduces that, for all $f \in C_{b}\left(D, \mathbb{R}_{+}\right)$,

$$
\widetilde{\eta}_{t}^{\infty} f=\widetilde{\eta}_{0}^{\infty} f+\int_{0}^{t} F\left(\widetilde{\eta}_{s}^{\infty}\right) f d s, \forall t \geq 0,
$$

which means that $\widetilde{\eta}^{\infty}$ is a weak solution to (3.13) and hence, by [2, Theorem 3.2], that $\widetilde{\eta}$ is an asymptotic pseudo-trajectory of the flow induced by (3.13).

\subsection{Proof of Theorem 2.1}

Fix any bounded measurable functions $f: D \rightarrow \mathbb{R}$. For all $n \geq 1$, we set

$$
\Psi_{n}=\theta_{(n+1)} \mu_{\theta_{(n+1)}} f-n \eta_{n} A f .
$$

The random sequence $\left(\Psi_{n}\right)_{n \geq 1}$ is a $\left(\mathcal{G}_{\ell}\right)_{\ell \geq 0}$-martingale and

$$
\Psi_{n}=\sum_{i=1}^{n} \int_{0}^{\tau_{\partial}^{(i+1)}} f\left(X_{s}^{(i+1)}\right) d s-\delta_{Z_{i}} A f .
$$

This martingale property implies that

$$
\begin{aligned}
\frac{\mathbb{E}\left[\left|\Psi_{n}\right|^{2}\right]}{n} & \leq \frac{1}{n} \sum_{i=1}^{n} \mathbb{E}\left[\left|\int_{0}^{\tau_{\partial}^{(i+1)}} f\left(X_{s}^{(i+1)}\right) d s-\delta_{Z_{i}} A f\right|^{2}\right] \\
& \leq 2\|f\|_{\infty}^{2} \sup _{x \in D} \mathbb{E}_{x}\left(\tau_{\partial}^{2}\right) .
\end{aligned}
$$


From [12, Theorem 1.3.17], we deduce that $n^{-1} \Psi_{n}$ goes almost surely to zero when $n$ goes to infinity, that is

$$
\frac{\theta_{(n+1)} \mu_{\theta_{n+1}} f}{n}-\eta_{n} A f \underset{n \rightarrow+\infty}{\stackrel{a . s .}{\longrightarrow}} 0 .
$$

Since $A f$ is continuous and bounded for any bounded measurable function $f$ (see Proposition 3.2), one deduces from Proposition 4.3 that, almost surely,

$$
\frac{\theta_{n} \mu_{\theta_{n}} f}{n} \underset{n \rightarrow+\infty}{\longrightarrow} \alpha A f=\alpha f / \lambda_{0} .
$$

Applying this result to $f=1_{D}$, one deduces that $\theta_{n} / n$ converges to $1 / \lambda_{0}$ almost surely and hence that $\mu_{\theta_{n}} f$ converges to $\alpha f$ almost surely. Since, for all $t \in\left[\theta_{n}, \theta_{n+1}\right)$,

$$
\begin{aligned}
\left|\mu_{t} f-\mu_{\theta_{n}} f\right| & \leq \frac{1}{t}\left|\int_{\theta_{n}}^{t} f\left(Y_{s}\right) d s\right|+\left|\int_{0}^{\theta_{n}} f\left(Y_{s}\right) d s\right|\left(\frac{1}{t}-\frac{1}{\theta_{n}}\right) \\
& \leq \frac{\|f\|_{\infty}}{t}\left(t-\theta_{n}\right)+\frac{\left(t-\theta_{n}\right)}{t \theta_{n}} \theta_{n}\|f\|_{\infty} \\
& \leq 2\|f\|_{\infty}\left(1-\frac{\theta_{n}}{\theta_{n+1}}\right)
\end{aligned}
$$

the almost sure convergence of $\mu_{t}$ to $\alpha f$ when $t \rightarrow+\infty$ follows from the almost sure convergence of $\theta_{n} / n$ to the positive constant $1 / \lambda_{0}$.

\section{References}

[1] D. Aldous, B. Flannery, and J. L. Palacios. Two applications of urn processes the fringe analysis of search trees and the simulation of quasi-stationary distributions of markov chains. Probab. Eng. Inf. Sci., 2(3):293-307, 1988.

[2] M. Benaïm. Dynamics of stochastic approximation algorithms. In Séminaire de Probabilités, XXXIII, volume 1709 of Lecture Notes in Math., pages 1-68. Springer, Berlin, 1999.

[3] M. Benaïm and B. Cloez. A stochastic approximation approach to quasistationary distributions on finite spaces. Electron. Commun. Prob., 20, 2015 .

[4] M. Benaïm, B. Cloez, F. Panloup, et al. Stochastic approximation of quasistationary distributions on compact spaces and applications. Ann. Appl. Probab., 28(4):2370-2416, 2018.

[5] M. Benaïm and M. W. Hirsch. Asymptotic pseudotrajectories and chain recurrent flows, with applications. J. Dynam. Differential Equations, 8(1):141-176, 1996. 
[6] J. Blanchet, P. Glynn, and S. Zheng. Analysis of a stochastic approximation algorithm for computing quasi-stationary distributions. Adv. Appl. Probab., 48(3):792-811, 2016.

[7] N. Champagnat, K. A. Coulibaly-Pasquier, and D. Villemonais. Criteria for exponential convergence to quasi-stationary distributions and applications to multi-dimensional diffusions. In Séminaire de Probabilités XLIX, pages 165-182. Springer, 2018.

[8] N. Champagnat and D. Villemonais. Exponential convergence to quasistationary distribution and Q-process. Probab. Theory Rel., 164(1):243$283,2016$.

[9] N. Champagnat and D. Villemonais. Uniform convergence of conditional distributions for absorbed one-dimensional diffusions. Adv. Appl. Probab., 50(1):178, 2018.

[10] N. Champagnat, D. Villemonais, et al. Uniform convergence to the $Q$ process. Electron. Comm. Prob., 22, 2017.

[11] P. Collet, S. Martínez, and J. Martín. Quasi-Stationary Distributions: Markov Chains, Diffusions and Dynamical Systems. Probability and Its Applications. Springer Berlin Heidelberg, 2012.

[12] M. Duflo. Random iterative models, volume 34 of Applications of Mathematics (New York). Springer-Verlag, Berlin, 1997. Translated from the 1990 French original by Stephen S. Wilson and revised by the author.

[13] S. N. Ethier and T. G. Kurtz. Markov processes. Characterization and convergence. Wiley Series in Probability and Mathematical Statistics: Probability and Mathematical Statistics. John Wiley \& Sons Inc., New York, 1986.

[14] C. Mailler and D. Villemonais. Stochastic approximation on non-compact measure spaces and application to measure-valued Pólya processes. To appear in Ann. Appl. Probab., 2018.

[15] S. Méléard and D. Villemonais. Quasi-stationary distributions and population processes. Probab. Surv., 9:340-410, 2012.

[16] M. Métivier and P. Priouret. Théorèmes de convergence presque sure pour une classe d'algorithmes stochastiques à pas décroissant. Probab. Theory Rel., 74(3):403-428, 1987.

[17] E. Priola and F.-Y. Wang. Gradient estimates for diffusion semigroups with singular coefficients. J. Funct. Anal., 236(1):244-264, 2006.

[18] H. Renlund. Generalized Polya urns via stochastic approximation. ArXiv e-prints, Feb. 2010. 
[19] E. A. van Doorn and P. K. Pollett. Quasi-stationary distributions for discrete-state models. European J. Oper. Res., 230(1):1-14, 2013.

[20] D. Villemonais. Interacting particle systems and Yaglom limit approximation of diffusions with unbounded drift. Electron. J. Probab., 16:no. 61, 1663-1692, 2011.

[21] A. Q. Wang, G. O. Roberts, and D. Steinsaltz. An approximation scheme for quasi-stationary distributions of killed diffusions. Stoch. Proc. Appl., 130(5):3193-3219, 2020. 